\title{
Supportive Information
}

\section{Atomic-level Drug Substance and Polymer Interaction in Posaconazole Amorphous Solid Dispersion from Solid-State NMR}

\author{
Xingyu Lu ${ }^{1, \S}$, Mingyue $\mathrm{Li}^{1, \S}$, Chengbin Huang ${ }^{1, \S}$, Michael B. Lowinger ${ }^{1}$, Wei Xu${ }^{1}$, Lian $\mathrm{Yu}^{2}$, Stephen R. \\ Byrn $^{3}$, Allen C. Templeton ${ }^{1}$, and Yongchao $\mathrm{Su}^{1,3,4^{*}}$ \\ ${ }^{1}$ Pharmaceutical Sciences, Merck \& Co., Inc., Kenilworth, NJ 07033, United States \\ ${ }^{2}$ School of Pharmacy and Department of Chemistry, University of Wisconsin-Madison, Madison, WI \\ 53705, United States \\ ${ }^{3}$ Department of Industrial and Physical Pharmacy, College of Pharmacy, Purdue University, Indiana \\ 47907, United States \\ ${ }^{4}$ Division of Molecular Pharmaceutics and Drug Delivery, College of Pharmacy, The University of Texas \\ at Austin, Austin, Texas 78712, United States
}

\section{Technical details of S-REDOR experiment}

The ${ }^{19} \mathrm{~F}$ carrier frequency was placed on resonance at $-112 \mathrm{ppm}$ to maximally invert the two fluorine signals. The RF field strength of SR $4_{1}^{2}$ recoupling scheme was $24 \mathrm{kHz}$. A ${ }^{13} \mathrm{C} 180$ degree pulse of $6.0 \mu \mathrm{s}$ was applied to refocus the ${ }^{13} \mathrm{C}$ chemical shift during the REDOR recoupling period. ${ }^{1} \mathrm{H}$ SPINAL64 decoupling was $120 \mathrm{kHz}$ during the REDOR period and $100 \mathrm{kHz}$ during acquisition to maximize ${ }^{13} \mathrm{C} \mathrm{T}_{2}$ and improve the spectral resolution. Full-echo spectra $S_{0}$ and dephased spectra $S$ were simultaneously acquired in each scan. $\mathrm{S}_{0}$ and $\mathrm{S}$ spectra at a series of recoupling times were obtained. In Figures 5A-C and S2, REDOR recouping times were 0.21 , 0.63, 1.04, 1.46, 1.88, 2.29, 2.71, 3.13, 3.54, 3.99, 4.38, 4.79 ms. RF inhomogeneity and ${ }^{19} \mathrm{~F}$ pulse imperfection may cause slight deviations of experimental curve from the ideal REDOR curve. The robustness of symmetry-based REDOR to rf inhomogeneity on symmetry-based REDOR has been explored ${ }^{1}$.

\section{Geometric calculation of two possible hydrogen bonds}

Two hydrogen bond patterns have been proposed in this study, including interactions between the O-H group of HPMCAS as an acceptor and POSA fluorine or difluorophenyl ring as a donor. To estimate these hydrogen bond distances, the following values are used: O-H covalent bond distance of $0.97 \AA$ (typical value for alcohols by neutron diffraction), ${ }^{2} \mathrm{C}-\mathrm{O}$ covalent bond distance of $1.42 \AA$ (extracted from D- 
mannitol crystal), ${ }^{3} \mathrm{C}-\mathrm{O}-\mathrm{H}$ angle of 110 degree (extracted from methanol crystal by neutron diffraction) ${ }^{4} \mathrm{C}$ $\mathrm{C}$ covalent bond distance of $1.38 \AA$ and $\mathrm{C}-\mathrm{F}$ covalent bond distance of $1.36 \AA .^{5}$

In the first scenario, fluorine is directly involved in hydrogen bond $\mathrm{O}-\mathrm{H} \cdots \mathrm{F}-\mathrm{C}$ between HPMCAS and POSA. Considering geometric impact of $\mathrm{sp}^{3}$ hybridization carbon, $\mathrm{C}_{\mathrm{OH}}$, which is connected to hydroxyl group in HPMCAS, the fluorine in POSA molecule is sterically hindered by other functional groups linked to $\mathrm{C}_{\mathrm{OH}}$. Theoretically, the $\mathrm{sp}^{3}$ hybridization carbon is a tetrahedral, resulting in the steric hindrance angle of 109.5 degree. In this study, we selected 109 degree as a boundary. Additionally, based on the literature, ${ }^{6}$ the $\mathrm{O}-\mathrm{H} \cdots \mathrm{F}$ angular $>90$ degree is a critical requirement for a meaningful hydrogen bond. Thus, we set the $\mathrm{O}-\mathrm{H} \cdots \mathrm{F}$ angular cutoff at $>90$ degree in the following calculations. On basis of the constant $\mathrm{C}_{\mathrm{OH}}-\mathrm{F}$ distance, a spherical coordinate system is established in Figure S3A. In this coordinate, carbon next to hydroxyl group in HPMCAS is the origin, oxygen of hydroxyl group in HPMCAS is along $z$ axis $(0 \AA, 0 \AA, 1.42 \AA)$ and hydrogen of hydroxyl group in HPMCAS is on the $x-z$ plane. The coordinate of hydrogen of hydroxyl group is $(0.912 \AA, 0 \AA, 1.752 \AA)$ and fluorine atom is $(r, \theta, \varphi)$ then, where $r$ is radial length of $\mathrm{C}_{\mathrm{OH}^{-}}-\mathrm{F}$ distance, $\theta$ polar angle and $\varphi$ azimuthal angle as shown in Figure S3A. When $\theta \leq 109$ degree, 0 degree $<\varphi$ $\leq 360$ degree, and $\mathrm{O}-\mathrm{H} \cdots \mathrm{F}$ angular $>90$ degree, the $\mathrm{H} \cdots \mathrm{F}$ hydrogen bond distance varies with $\theta$ and $\varphi$, and ranges from $2.7 \AA$ to $5.9 \AA\left(\mathrm{C}_{\mathrm{OH}^{-}}-\mathrm{F}\right.$ distance of $\left.4.3 \AA\right)$ as shown in Figure 10A. In the situation of $\mathrm{C}_{\mathrm{OH}^{-}}-\mathrm{F}$ distance $4.5 \AA$, the $\mathrm{H} \cdots \mathrm{F}$ hydrogen bond distance varies from $2.9 \AA$ to $6.1 \AA$.

Another possible configuration is the formation of hydrogen bond between hydroxyl group in HPMCAS and phenyl group in posaconazole. Herein, we consider the bifluorophenyl group as a plane and the center of phenyl plane is represented by $\mathrm{M}$ in Figure S3B. In this configuration, the hydroxyl group is directional to the center of phenyl group to simplify the calculation. In the established three-dimensional coordinate system (Figure S3B), the center of phenyl group, M, is the origin and the bifluorophenyl plane is on the $x-y$ plane. Hydrogen and oxygen atoms of hydroxyl group in HPMCAS are along $z$ axis. The carbon next to hydroxyl group in HPMCAS is constrained by C-O distance of $1.42 \AA$ and C-O-H angle of 110 degree, limiting its position on a circle (the center of such circle is on the $z$ axis). Here, we defined an angle between the projection of C-O bond on the phenyl plane ( $x-y$ plane) and C-F bond in phenyl group as $\alpha$. By varying the angle of $\alpha$, the bond distance of $\mathrm{H} \cdots \mathrm{M}$ is within $2.6 \AA$ when $\mathrm{C}_{\mathrm{OH}^{-}}-\mathrm{F}$ distance of $4.3 \AA$ in Figure 10B. The bond distance of $\mathrm{H} \cdots \mathrm{M}$ is up to $2.8 \AA$ if $\mathrm{C}_{\mathrm{OH}^{-}} \mathrm{F}$ distance is $4.5 \AA$. Since this calculation only considers mathematical geometries and no chemical rationales was involved, Figure S3B includes some impossible distances, e.g. $\leq 0 \AA$ at $\alpha$ of $180^{\circ}$. 


\section{Thermal analysis}

The thermogravimetric analysis (TGA) was performed in a TA Instruments TGA Q5000. A heating rate of $20 \mathrm{~K} / \mathrm{min}$ and $100 \mathrm{~mL} / \mathrm{min} \mathrm{N}_{2}$ purge were utilized. The differential scanning calorimetry (DSC) plot in Figure S4 was reproduced from our previous study. ${ }^{7}$ Briefly, DSC was performed in hermetic aluminum pans using a TA Instruments DSC Q2000. 5-10 mg of samples was packed into a hermetically sealed aluminum pan. The samples were then cooled and heated at $10 \mathrm{~K} / \mathrm{min}$ to determine the glass transition temperature $\left(T_{\mathrm{g}}\right)$ under $50 \mathrm{~mL} / \mathrm{min}_{2}$ purge. The reported $T_{\mathrm{g}}$ is the onset temperature of the glass transition.

Table S1. ${ }^{13} \mathrm{C}$ chemical shift assignments of POSA amorphous reference ${ }^{8}$ and POSA-HPMCAS amorphous solid dispersion.

\begin{tabular}{|c|c|c|}
\hline${ }^{13} \mathrm{C}$ No. & $\begin{array}{l}\text { Amorphous } \\
\text { reference }^{8}\end{array}$ & $\begin{array}{c}\text { Amorphous Solid } \\
\text { Dispersion }\end{array}$ \\
\hline 3 & 39.7 & 39.9 \\
\hline 8 & 151.5 & 150.6 \\
\hline $9 *$ & 114.5 & 117.7 \\
\hline $10 *$ & 116.7 & 117.7 \\
\hline 11 & 146.3 & 145.9 \\
\hline $12 *$ & 116.7 & 117.7 \\
\hline $13 *$ & 114.5 & 117.7 \\
\hline 14 & 127.0 & 126.7 \\
\hline 15 & 159.2 & 159.5 \\
\hline 17 & 163.5 & 163.1 \\
\hline 18 & 111.9 & 112.5 \\
\hline 19 & 127.5 & 126.7 \\
\hline 23 & 151.1 & 150.6 \\
\hline 25 & 145.8 & 145.9 \\
\hline 27 & 49.8 & 51.7 \\
\hline 28 & 49.8 & 49.7 \\
\hline 30 & 49.8 & 49.7 \\
\hline 31 & 49.8 & 51.7 \\
\hline 34 & 151.0 & 150.6 \\
\hline $35^{*}$ & 116.7 & 117.7 \\
\hline $36^{*}$ & 122.1 & 117.7 \\
\hline 37 & 127.0 & 126.7 \\
\hline $38^{*}$ & 122.1 & 117.7 \\
\hline $39 *$ & 116.7 & 117.7 \\
\hline 41 & 153.4 & 153.3 \\
\hline 44 & 134.9 & 134.8 \\
\hline 50 & 11.1 & 11.0 \\
\hline
\end{tabular}

*Tentatively assigned due to peak overlapping 


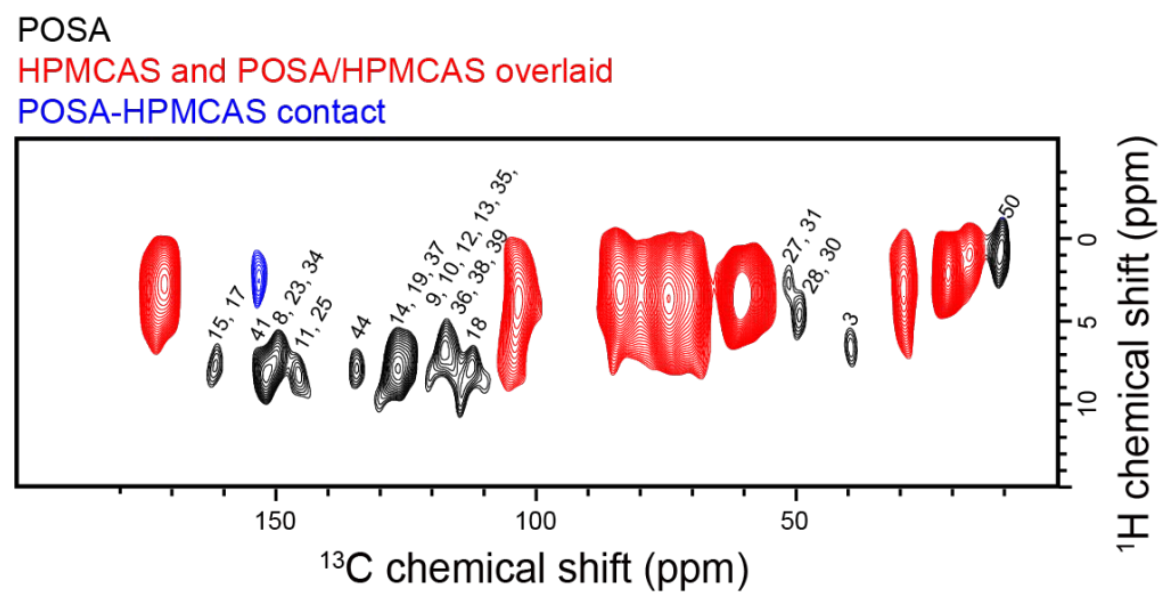

Figure S1. 2D ${ }^{1} \mathrm{H}-{ }^{13} \mathrm{C}$ HETCOR spectrum of POSA-HPMCAS ASDs at 30\% drug loading. The spectrum is colored according to the origin of the peaks as indicated. Chemical shift assignments of POSA in the ASD are labeled on the spectrum. The figure was reproduced from our previous study. ${ }^{7}$
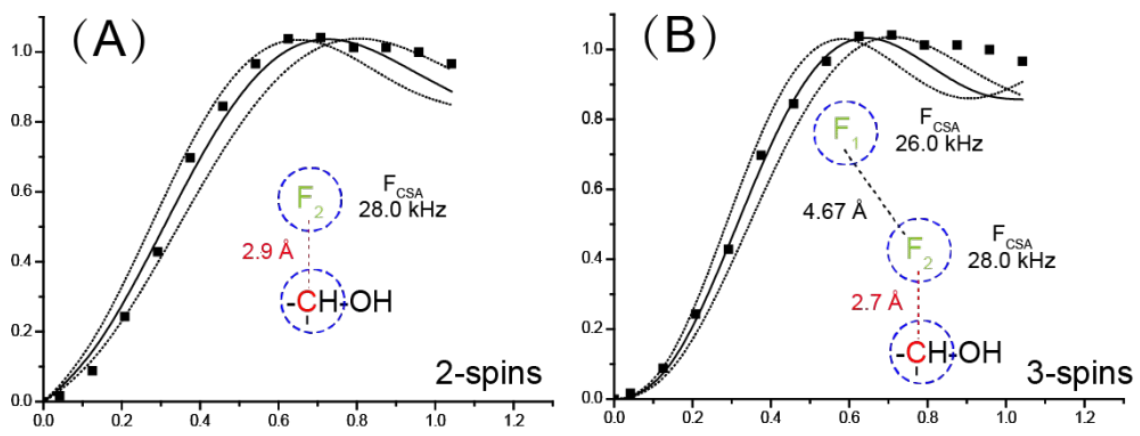

Figure S2. Quantitative distances in crystalline POSA measured by ${ }^{13} \mathrm{C}-{ }^{19} \mathrm{~F}$ REDOR using (A) two-spin and (B) three-spin system fittings for intramolecular contacts of C5-F2. 

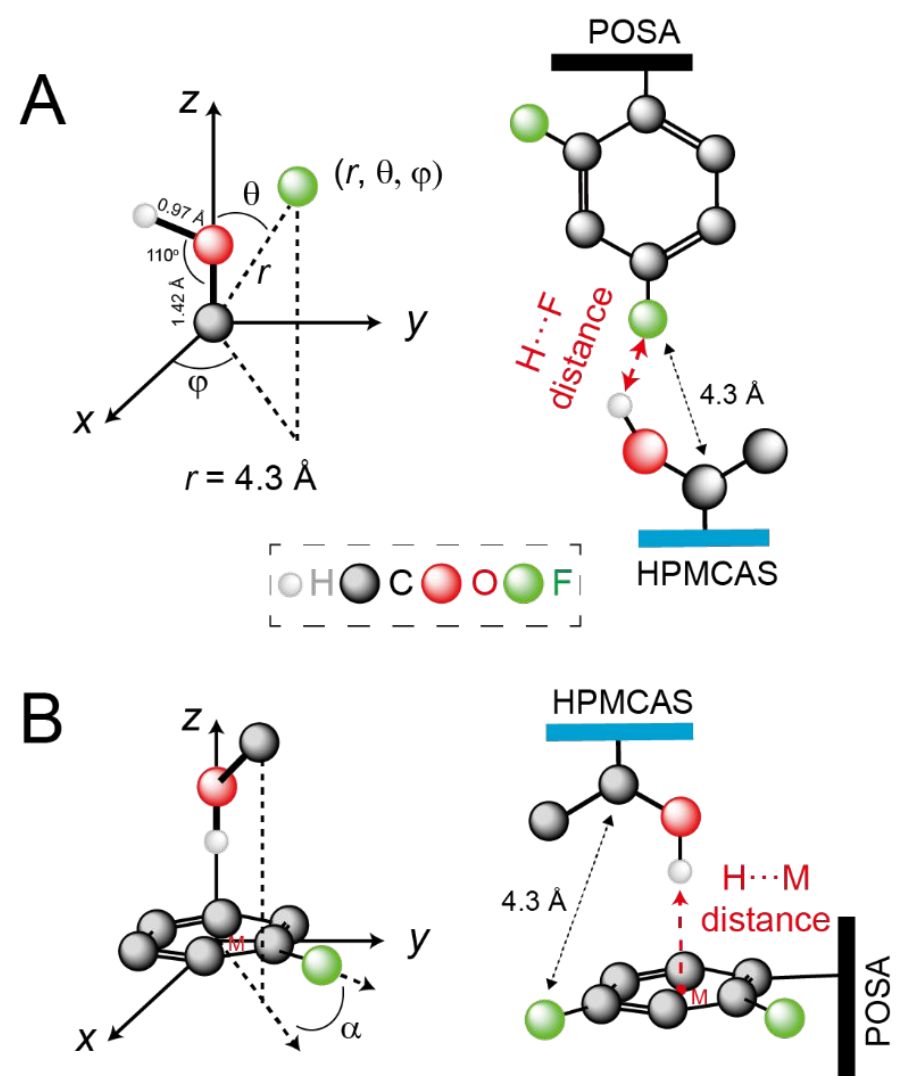

Figure S3. Schematic diagrams of geometric calculation for two hypothetic hydrogen bonding complexes formed between POSA and HPMCA including interaction between the polymer O-H group as an acceptor and POSA fluorine (A) or difluorophenyl ring (B) as a donor. 

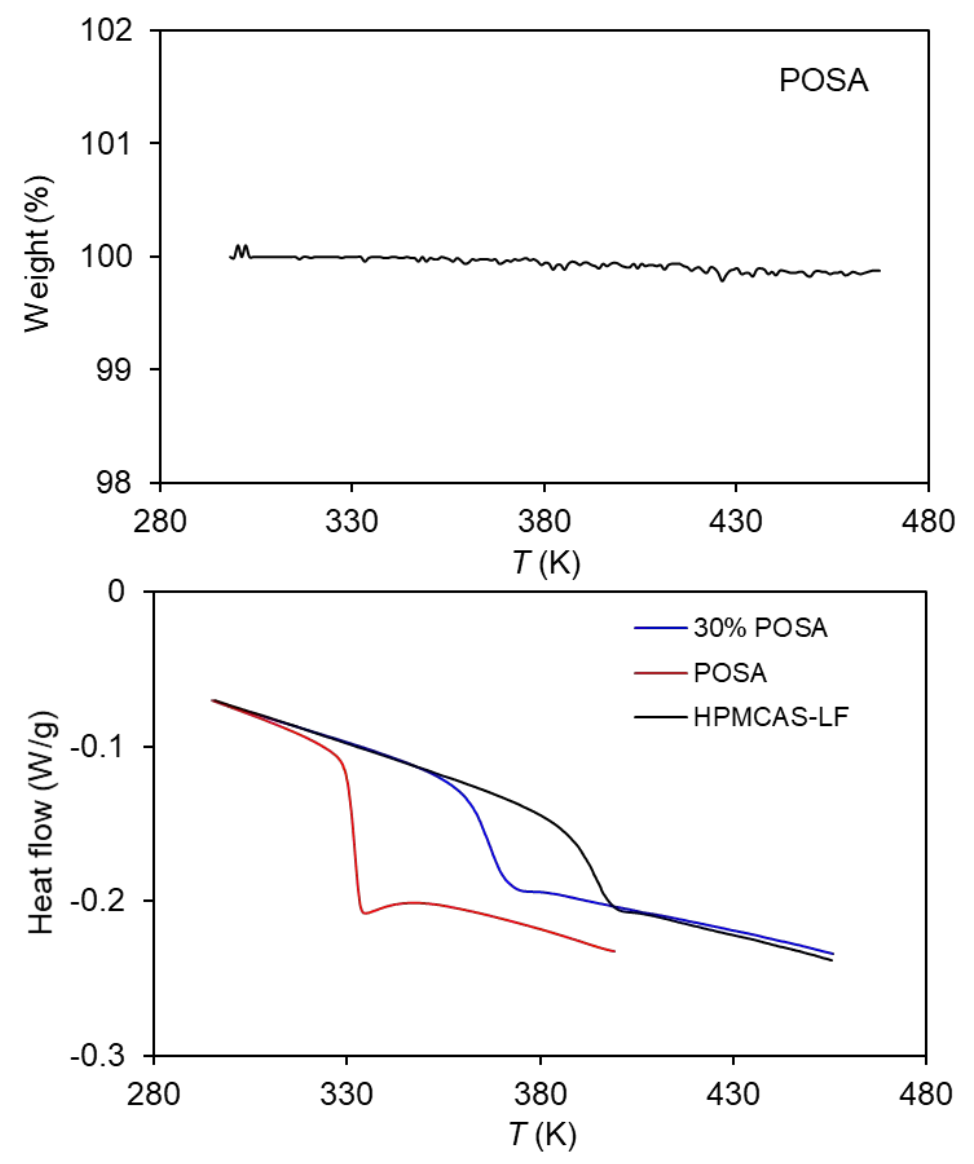

Figure S4. Top: thermogravimetric analysis of POSA; and bottom: DSC traces of the glass transition event in melt-quenched POSA, HPMCAS and POSA-HPMCAS dispersion. ${ }^{7}$ 


\section{References:}

1. Chen, L.; Wang, Q. A.; Hu, B. W.; Lafon, O.; Trebosc, J.; Deng, F.; Amoureux, J. P., Measurement of hetero-nuclear distances using a symmetry-based pulse sequence in solid-state NMR. Physical Chemistry Chemical Physics 2010, 12 (32), 9395-9405.

2. Allen, F. H.; Kennard, O.; Watson, D. G.; Brammer, L.; Orpen, A. G.; Taylor, R., Tables of bond lengths determined by X-ray and neutron diffraction. Part 1. Bond lengths in organic compounds. Journal of the Chemical Society, Perkin Transactions 2 1987, (12), S1-S19.

3. Fronczek, F. R.; Kamel, H. N.; Slattery, M., Three polymorphs ([alpha], [beta], and [delta]) of dmannitol at 100 K. Acta Crystallographica Section C 2003, 59 (10), o567-o570.

4. Torrie, B. H.; Weng, S. X.; Powell, B. M., Structure of the $\alpha$-phase of solid methanol. Molecular Physics 1989, 67 (3), 575-581.

5. McQuiston, D. K.; Mucalo, M. R.; Saunders, G. C., The structure of posaconazole and its solvates with methanol, and dioxane and water: Difluorophenyl as a hydrogen bond donor. Journal Of Molecular Structure 2019, 1179, 477-486.

6. Steiner, T., The Hydrogen Bond in the Solid State. Angewandte Chemie International Edition 2002, $41(1), 48-76$.

7. Huang, C.; Klinzing, G.; Procopio, A.; Yang, F.; Ren, J.; Burlage, R.; Zhu, L.; Su, Y. Understanding compression-induced amorphization of crystalline posaconazole. Mol. Pharm. 2019, 16 (2), 825-833.

8. $\quad$ Lu, X.; Huang, C.; Li, M.; Skomski, D.; Xu, W.; Yu, L.; Byrn, R. S.; Templeton, C. A.; Su, Y., Molecular Mechanism of Crystalline-to-Amorphous Conversion of Pharmaceutical Solids from ${ }^{19} \mathrm{~F}$ Magic Angle Spinning NMR. J. Phys. Chem. B, epub online, 2020. https://doi.org/10.1021/acs.jpcb.0c02131 